\title{
UCS intensity and eyelid conditioning: Another look
}

The psychological literature concerning UCS intensity and eyelid conditioning is briefly reviewed. It is argued, contrary to Spence and Platt, that this literature does not at present contain convincing data to support the conclusion that there are significant effects of UCS intensity upon performance.

In a recent article, Spence \& Platt (1966) conclude that the evidence they present "overwhelmingly refutes" Burstein's (1965a) suggestion in this Journal that UCS intensity may not affect asymptotic performance in eyelid conditioning, but may determine instead whether or not individual Ss condition.

The implication of Spence and Platt's article is that significant UCS intensity effects exist even when nonconditioners (NCs) are removed following Burstein's procedure. Burstein, however, defined as NCs Ss "who did not average over one CR per block of 10 trials for the first 50 trials," while Spence and Platt classified as NCs "any S who made less than $10 \%$ CRs in the trial blocks being analyzed." In the absence of any data or rationale supporting the interchangeability of these criteria, Spence and Platt's use of the last 20,40 , or 50 trials makes any comparison with Burstein's results difficult if not impossible.

Spence and Platt contend that even if removing NCs did result in equivalent performance from groups conditioned with different UCS intensities, the admissability of comparing such groups is questionable, since "assuming the distribution of conditionability of Ss, i.e., their capacity to be conditioned, is the same in the original groups, it places one in the position of comparing subsamples in which this is no longer the case." However, while they introduce "conditionability" to describe the distribution of capacity for conditioning, they later employ it with respect to the performance distribution from which NCs are removed. As Spence and Platt acknowledge, the frequency of NCs is typically greater in low UCS groups; therefore, these performance differences cannot be attributed to differences in the capacity to be conditioned-but are instead, as suggested by Burstein, clearly generated by the UCS intensities employed in the conditioning procedures.

Several of the Iowa studies cited by Spence and Platt involve Ss selected on the basis of extreme scores on the TMAS。 That using such Ss is a complicating factor can be seen from the following comment: "The shape of the conditioning curves suggests that the anxious Ss had reached asymptote...by the end of the $(80)$ trials, whereas the curve for the non-anxious
Ss was still showing an increase" (Spence \& Taylor, 1951). While Spence and Platt report no UCS Anxiety interaction, this finding does not extend to unsampled populations, i.e., to those Ss who do not fall in the highest and lowest pentiles on the TMAS.

A study by Gormezano et al (1962) reporting no differences as a function of UCS intensity is termed "irrelevant" by Spence and Platt because, "as UCS intensity increased, the performance level of avoidance Ss increased so that their yoked classical controls received fewer reinforcements," producing "an inverse ordering of the classical groups on the UCS dimension." However, the published curves reveal that on six of the seven trial blocks plotted there is no inverse ordering of the classical groups in relation to the avoidance groups. Moreover, the highest performance in the avoidance groups is that of the intermediate UCS group and not the high UCS group. Thus, the assertion that the classical groups received fewer and fewer reinforcements as UCS intensity increased seems unsupported, and Gormezano et al's conclusion that "the UCS dimension failed to produce significant differences in either acquisition or extinction" seems to be warranted. Spence and Platt apparently overlooked Runquist's (1963) report that, with groups receiving the same $(50 \%)$ partial reinforcement schedule, no difference in performance was obtained as a function of UCS intensity.

A study by Beck (1963) is cited by Spence and Platt because the difference in performance of groups conditioned with high and low UCS intensities yielded such a high F-ratio that, they say, it is unlikely that the removal of NCs would reduce it to nonsignificance. It seems possible that there was something unusual about Beck's procedures, since, while the majority of eyelid investigators have reported no effect of CS intensity, Beck reports an F-ratio of 204.07 for this variable. Moreover, although Beck attempted to correct for voluntary responders by adjusting her data on the basis of response form, she did not discard any Ss as voluntary responders. The comment of another eyelid conditioner is of interest: "Women were not used when it was found that a very high proportion of them responded with voluntary blinks to the CS under the condition in which the UCS (air puff) was very strong $(5.00 \mathrm{lb} . / \mathrm{sq}$. in.)" (Spence, 1953). Beck used $152 \mathrm{Ss,}$ all of whom were women, and a high UCS intensity of $5.00 \mathrm{psi}$. Since voluntary responses were so numerous under these conditions that Spence was forced to discard all women, 
it would be interesting to see what the effect would be of eliminating both voluntary responders and nonconditioners from Beck's data. It is of interest, also, that in the cited experiments of Spence, Haggard, \& Ross (1958), Runquist, Spence, \& Stubbs (1958) and Spence \& Tandler (1963) all Ss were women.

Spence and Platt introduce the results of Trapold \& Spence (1960) by saying that "according to Burstein this reduction (in the reinforcing UCS intensity) should have had no effect on the performance level of this group." They go on to point out that the UCS reduction resulted instead in a decrease in performance level and to assert that this decrease "cannot be ascribed in any way to nonconditioners."

Disregarding the fact that, along with several other of the cited studies, Trapold and Spence's procedure involved the use of interpolated unpaired UCSs-a controversial manipulation assumed by some (Kimble, Mann, \& Dufort, 1955) to elevate drive, and thus response level, although this assumption has received no support in other studies, e.g., Goodrich, Ross, \& Wagner, 1957; Loess, 1964-Spence and Platt seem not entirely clear as to the implications of Burstein's results. First, they attribute a prediction to Burstein which is invalidated by his published data and secondly, they contend that the decrease in performance cannot be attributed to NCs in spite of the evidence in Burstein's study that such performance decrements are eliminated when NCs are removed from the data. It is unfortunate that Spence and Platt did not provide some empirical basis for their statement by removing NCs from Trapold and Spence's data.

In the one study (Spence, 1953) reported by Spence and Platt in which NCs were defined following Burstein's procedure, significant differences are reported for both the pre- and post-shift phases whether or not NCs are removed. However, nothing approaching asymptotic performance was obtained in the preshift performance, the low UCS group performing at about $17 \%$ CRs and the high UCS group at about $35 \%$ CRs. That the 30 trials allowed by Spence were not sufficient to permit asymptotic performance is quite evident from the postshift performance of those Ss whose conditioning was continued without a change in UCS intensity.

In this study, as originally published, the statistical analysis was performed on the first 20 postshift trials, rather than on the 70 postshift trials analyzed by Spence and Platt, because "score distributions for longer periods were quite unsatisfactory" (Spence, 1953). It is difficult to understand, therefore, how a statistical analysis which was said to be inappropriate at that time can become appropriate at the present time.

Another point of interest concerns those studies cited by Spence and Platt which involve large Ns (100 or more). One of these (Spence \& Taylor, 1951) failed to obtain a significant effect of UCS intensity with or without nonconditioners removed. Two others (Spence, Haggard, \& Ross, 1958; Spence, 1958) are revealing in that removal of nonconditioners reduces the significance level from .001 to .05 . It would be interesting to know what the effect would be of substituting the $10 \%$ NC criterion used by Burstein for the less than 10\% NC criterion used by Spence and Platt.

It might also be pointed out that the suggestion of Burstein handles rather easily those studies which do not report a performance difference as a function of UCS intensity (e.g., Spence \& Taylor, 1951; Gormezano et al, 1962; Runquist, 1963; Burstein, 1965b) by assuming that specific aspects of the experimental situation or sampling error have resulted in equal numbers of $\mathrm{NCs}$ in different UCS groups, an assumption which is capable of empirical verification. Although Spence and Platt argue that such studies have used asymptotic UCS values, this seems questionable in view of the significant differences reported by Burstein prior to the removal of NCs. Spence and Platt also seem to be inconsistent in assuming that Burstein's UCS values (.75 and 5.5 psi) are asymptotic while Passey's (.75 and $8.8 \mathrm{psi}$ ) are not.

In conclusion, it should be made very clear that these comments are not intended to discount the possibility that significant effects of UCS intensity upon performance level could exist after nonconditioners are removed from the data, but are designed merely to point out that convincing data supporting such a conclusion are, at the present time, not a part of the psychological literature. Moreover, with the mounting number of studies in the literature demonstrating the critical roles played by such variables as instructions, sex, attitudes, ready signals, anxiety, etc., in determining performance, it would seem that we are not ready to construct a definitive picture of the relationship between UCS intensity and performance.

\section{References ${ }^{1}$}

Burstein, K. R. Effect of UCS intensity upon the acquisition of conditioned responses acquired under a lengthened interstimulus interval. J. exp. Psychol., 1965b, 70, 147-151.

Goodrich, K. P., Ross, L. E., \& Wagner, A. R. Performance in eyelid conditioning following interpolated presentations of the UCS. J. exp. Psychol., 1957, 53, 214-217.

Kimble, G. A., Mann, L. I., \& Dufort, R. H. Classical and instrumental eyelid conditioning. J. exp. Psychol., 1955, 49, 407-417.

Loess, H. Effect of unpaired UCS presentations on performance in eyelid conditioning. Psychol. Rep., 1964, 15, 339348.

Runquist, $\boldsymbol{W}$. $\mathbf{N}$. Performance in eyelid conditioning following changes in reinforcement schedule, J. exp. Psychol., 1963, 65, 417-419.

Spence, K. W., \& Platt, J. R. UCS intensity and performance in eyelid conditioning. Psychol. Bull., 1966, 65, 1-10.

\section{Note}

1. Because of space limitations only those studies not cited by Spence and Platt are listed. 